\title{
Computing the distribution of the product of two continuous random variables
}

\author{
Andrew G. Glen ${ }^{\mathrm{a}}$, Lawrence M. Leemis ${ }^{\mathrm{b}, *}$, John H. Drew ${ }^{\mathrm{b}}$ \\ a Department of Mathematical Sciences, The United States Military Academy, West Point, \\ NY 10996, USA \\ ${ }^{\mathrm{b}}$ Department of Mathematics, The College of William and Mary, P.O. Box 8795, Williamsburg, \\ VA 23187-8795, USA
}

Received 1 September 2001; received in revised form 1 July 2002

\begin{abstract}
We present an algorithm for computing the probability density function of the product of two independent random variables, along with an implementation of the algorithm in a computer algebra system. We combine this algorithm with the earlier work on transformations of random variables to create an automated algorithm for convolutions of random variables. Some examples demonstrate the algorithm's application.
\end{abstract}

(C) 2002 Elsevier B.V. All rights reserved.

Keywords: Algorithms; Computational algebra systems; Convolutions; Probability

\section{Introduction}

Rohatgi's well-known result (1976, p. 141) for determining the distribution of the product of two random variables is straightforward to derive, but difficult to implement. Let $X$ and $Y$ be continuous random variables with joint PDF $f_{X, Y}(x, y)$. The PDF of $V=X Y$ is

$$
f_{V}(v)=\int_{-\infty}^{\infty} f_{X, Y}\left(x, \frac{v}{x}\right) \frac{1}{|x|} \mathrm{d} x
$$

\footnotetext{
* Corresponding author.

E-mail addresses: aa1275@usma.edu (A.G. Glen), leemis@math.wm.edu (L.M. Leemis), jhdrew@math.wm.edu (J.H. Drew).
} 
The implementation of this result, however, is not straightforward for general $X$ and $Y$. Springer (1979) presents a chapter on finding distributions of products of random variables, relying mostly on Laplace and Mellin transformation techniques, as implementation of the earlier result is often too cumbersome. Difficulties occur as a result of both the myriad of variations to the limits of integration and the propensity of the PDF of $V$ to be defined in a piece-wise manner. In this paper, we consider the cases when $X$ and $Y$ are independent and may have probability density functions (PDFs) defined in a piece-wise fashion. This simplification implies that the joint PDF of $X$ and $Y$ will be defined on a rectangular product space, which can be easily broken into special cases based on geometry. This special case of Rohatgi's result is implemented in a computer algebra system by enumerating all possible sets of limits of integration. We extend the use of this algorithm for products of independent random variables to create an algorithm for convolutions of independent random variables. These algorithms and their implementations are important procedures in A Probability Programming Language (APPL, Glen et al., 2001), a Maple-based software package (Maple, 2001), whose purpose is to automate operations on random variables.

\section{Theorem}

Considering a special case of Rohatgi's result will illustrate some of the issues associated with a general algorithm for determining the PDF of the product of two independent random variables. For completeness, the theorem is proven from first principles (using the transformation technique) even though it could be stated that it is a special case of Rohatgi's result. Assume that the random variable $X$ has support on the interval $(a, b)$ and the random variable $Y$ has support on the interval $(c, d)$. Also, the product space of the two random variables is assumed to fall entirely in the first quadrant. Theorems and proofs for other rectangular support regions are similar. The algorithm described in Section 3 includes all possible scenarios.

Theorem. Let $X$ be a random variable of the continuous type with PDF $f(x)$ which is defined and positive on the interval $(a, b)$, where $0<a<b<\infty$. Similarly, let $Y$ be a random variable of the continuous type with PDF $g(y)$ which is defined and positive on the interval $(c, d)$, where $0<c<d<\infty$. The PDF of $V=X Y$ is

$$
h(v)= \begin{cases}\int_{a}^{v / c} g\left(\frac{v}{x}\right) f(x) \frac{1}{x} \mathrm{~d} x, & a c<v<a d, \\ \int_{v / d}^{v / c} g\left(\frac{v}{x}\right) f(x) \frac{1}{x} \mathrm{~d} x, & a d<v<b c, \\ \int_{v / d}^{b} g\left(\frac{v}{x}\right) f(x) \frac{1}{x} \mathrm{~d} x, & b c<v<b d,\end{cases}
$$


when $a d<b c$,

$$
h(v)= \begin{cases}\int_{a}^{v / c} g\left(\frac{v}{x}\right) f(x) \frac{1}{x} \mathrm{~d} x, & a c<v<a d, \\ \int_{v / d}^{b} g\left(\frac{v}{x}\right) f(x) \frac{1}{x} \mathrm{~d} x, & a d<v<b d,\end{cases}
$$

when $a d=b c$, and

$$
h(v)= \begin{cases}\int_{a}^{v / c} g\left(\frac{v}{x}\right) f(x) \frac{1}{x} \mathrm{~d} x, & a c<v<b c, \\ \int_{a}^{b} g\left(\frac{v}{x}\right) f(x) \frac{1}{x} \mathrm{~d} x, & b c<v<a d, \\ \int_{v / d}^{b} g\left(\frac{v}{x}\right) f(x) \frac{1}{x} \mathrm{~d} x, & a d<v<b d,\end{cases}
$$

when $a d>b c$.

Proof. Only the case of $a d<b c$ is considered. The other cases are proven analogously. Using the transformation technique (Hogg and Craig, 1995, p. 173), the transformation $Z=X$ and $V=X Y$ constitutes a $1-1$ mapping from $\mathscr{A}=\{(x, y) \mid a<x<b, c<y<d\}$ to $\mathscr{B}=\{(z, v) \mid a<z<b, c z<v<\mathrm{d} z\}$. Let $u$ denote the transformation and $w$ the inverse transformation. The transformation, inverse, and Jacobian are:

$$
\begin{aligned}
& z=u_{1}(x, y)=x, \quad x=w_{1}(z, v)=z, \\
& v=u_{2}(x, y)=x y, \quad y=w_{2}(z, v)=v / z, \\
& J=\left|\begin{array}{cc}
1 & 0 \\
-v / z^{2} & 1 / z
\end{array}\right|=1 / z .
\end{aligned}
$$

The joint PDF of $Z$ and $V$ is

$$
f_{Z, V}(z, v)=f\left(w_{1}(z, v)\right) g\left(w_{2}(z, v)\right)|J|, \quad(z, v) \in \mathscr{B}
$$

or

$$
f_{Z, V}(z, v)=f(z) g(v / z) \frac{1}{|z|}, \quad(z, v) \in \mathscr{B} .
$$

Integrating with respect to $z$ over the appropriate intervals and replacing $z$ with $x$ in the final result yields the marginal PDF of $V$

$$
h(v)= \begin{cases}\int_{a}^{v / c} g\left(\frac{v}{x}\right) f(x) \frac{1}{x} \mathrm{~d} x, & a c<v<a d, \\ \int_{v / d}^{v / c} g\left(\frac{v}{x}\right) f(x) \frac{1}{x} \mathrm{~d} x, & a d<v<b c, \\ \int_{v / d}^{b} g\left(\frac{v}{x}\right) f(x) \frac{1}{x} \mathrm{~d} x, & b c<v<b d,\end{cases}
$$

as desired. 


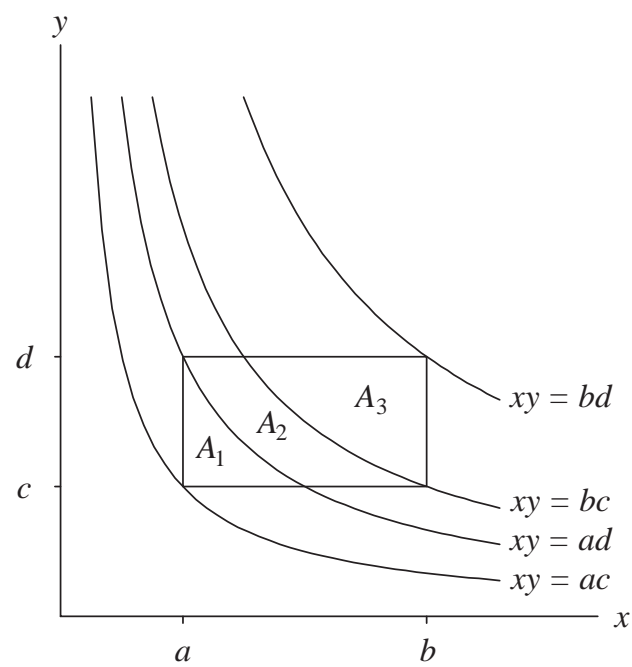

Fig. 1. The support of $X$ and $Y$ when $a d<b c$.

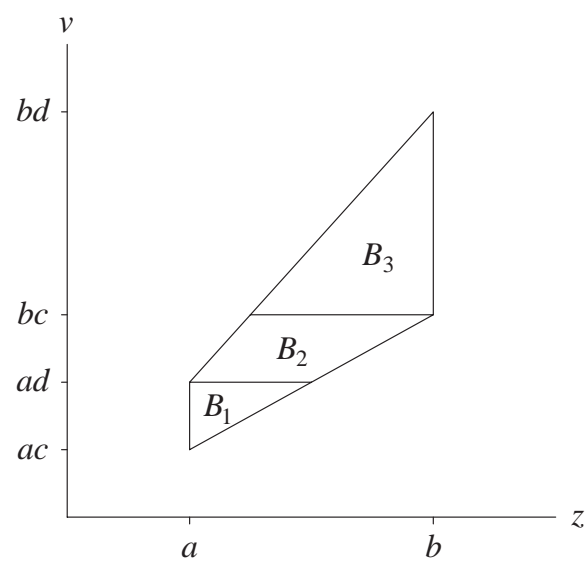

Fig. 2. The mapping of $Z=X$ and $V=X Y$ when $a d<b c$.

The geometry associated with the transformation for the case $a d<b c$ considered in the proof is shown in Figs. 1 and 2. The transformation maps $A_{i}$ onto $B_{i}$ for $i=1,2,3$. Although the transformation technique has been used to prove this theorem, the cdf technique could also have been used. The theorem also follows directly from Rohatgi's result since $f_{X, Y}(x, v / x)=f(x) g(v / x)$, which is non-zero only when $a<x<b$ and $c<v / x<d$. Thus $\max \{a, v / d\}<x<\min \{b, v / c\}$, which yields the limits of integration displayed in the theorem. The geometry of the case $a d<b c$ is shown in Fig. 1 in which the regions $A_{1}, A_{2}$ and $A_{3}$ correspond to the three different integrals for that 
case. Each $A_{i}$ corresponds to a different interval of $v$ for the family of hyperbolas given by $x y=v$. Given $v$, the interval of integration consists of those values of $x$ for which the curve $x y=v$ intersects the rectangle $\{(x, y): a<x<b, c<y<d\}$.

\section{Algorithm development}

The theorem in the previous section illustrates the importance of considering the magnitudes of the product of the coordinates of the southeast and northwest corners of the product space [i.e., $(b, c)$ and $(a, d)]$ when it lies entirely in the first quadrant. In order to apply the theorem to any continuous random variables $X$ and $Y$, three generalizations need to be addressed.

(1) Analogous theorems must be written for the cases when the $(a, b)$ by $(c, d)$ rectangle lies wholly in one of the other three quadrants. Rectangles that lie in more than one quadrant can be split into parts.

(2) Instead of having PDFs that are specified by a single standard function over their entire support, the random variables $X$ and $Y$ may be defined in a piece-wise manner over several intervals, forming many segments to the PDF (e.g., the triangular distribution).

(3) The cases when 0 and/or $\pm \infty$ belong to the endpoints of the intervals which constitute the support of $X$ and $Y$ must be considered (e.g., the normal distribution, see Examples 4.3 and 4.4).

These generalizations result in 24 ( 6 for each quadrant) primary cases that must be considered in order to correctly compute the limits of integration of the theorem. Further, some of these 24 cases must be broken into as many as 3 subcases, as illustrated in the theorem. In the first quadrant (i.e., $a>0$ and $c>0$ ), for example, we must consider the primary cases:

(1) $c=0$ and $d=\infty$ (Scenario A from the algorithm given in the appendix),

(2) $c=0$ and $d<\infty$ (Scenario B),

(3) $c>0$ and $d=\infty$ (Scenario C),

(4) $c>0$ and $d<\infty$ (Scenario D, with Case I: $a d<b c$ ),

(5) $c>0$ and $d<\infty$ (Scenario D, with Case II: $a d=b c$ ), and

(6) $c>0$ and $d<\infty$ (Scenario D, with Case III: $a d>b c$ ).

The paragraphs below provide more details. For quadrants II, III and IV, the limits of integration must also be set based on the appropriate geometry, as in Fig. 1.

For random variables that are defined in a piece-wise fashion over various intervals, let $n$ be the number of intervals for $X$ and let $m$ be the number of intervals for $Y$. There are $m n$ rectangular "product spaces" and the contribution of each to the value of the PDF of $V=X Y$ must be computed. Furthermore, each "product space" can contribute differently to the PDF of $V$ on up to three segments of the support of $V$ (see the theorem). As a result, the PDF of $V$ tends to become complicated very quickly, with an upper limit of $3 m n$ segments to its PDF. Furthermore, each of these segments fits 
into one of the 24 cases of the integration mentioned above. For example, the product of two $U(0,1)$ random variables yields a random variable $V$ with only one segment. But with only a slight change, e.g., $X \sim U(1,2)$ and $Y \sim U(3,4)$, yields a $V=X Y$ defined differently on three segments (see Example 4.1).

The case where the support of a random variable contains 0 (e.g., $U(-1,2)$ ) poses special difficulty since some of the rectangular product spaces will not lie wholly in any one quadrant and cannot be handled by the previously developed techniques. Our solution to this difficulty is to artificially add 0 as one of the endpoints of the intervals for $X$ and $Y$ whenever this case occurs, producing redundant segments, i.e., two segments on either side of zero with the same formula for the PDF. This method allows for implementation when the support area crosses into more than one quadrant. Example 4.3 gives an application of this special case, where the area of interest lies in all four quadrants. The algorithm subdivides the total area into four components, each entirely in one quadrant, and calculates the relevant portion of the new PDF for each quadrant.

The algorithm consists of a set-up portion, followed by nested loops that determine the contribution to the PDF of $V=X Y$ separately for each of the four quadrants. The appendix contains the set-up portion and the algorithm associated with the first quadrant. The algorithm for the other quadrants is similar.

The set-up phase begins by setting $n$ and $m$ to the number of intervals that form the support of $X$ and $Y$. Next, 0 is added as an interval delimiter for $X$ and/or $Y$ if the random variable can assume both positive and negative values, and 0 is not already an interval delimeter. Finally, the endpoints of the intervals that form the support of $V$ are determined by taking all products of the endpoints of the $X$ intervals times the endpoints of the $Y$ intervals.

A nested set of loops follows that treats all pairings of $X$ and $Y$ intervals. As shown in Fig. 1, the coordinates $(a, c)$ are assigned to the southwest corner of the current rectangle of interest, and the coordinates $(b, d)$ are assigned to the northeast corner of the current rectangle of interest. A test to determine which quadrant contains the current rectangle is made at this point. Adding 0 as an interval delimiter in the set-up phase assures that the current rectangle will be completely contained in just one of the quadrants. Once the quadrant is determined, tests on $c$ and $d$ determine which integrals should be computed and the appropriate limits of integration. Finally, the insertion of 0 sometimes leads to a PDF for $V$ with the same formula on both sides of 0 . If this occurs, the program simplifies the PDF by removing 0 as an interval endpoint if the function is defined at 0 .

\section{Examples}

This section contains applications of using the APPL procedure Product, which is our implementation of the algorithm described in the previous section.

Example 4.1. Consider the random variable $X \sim U(1,2)$ and the random variable $Y \sim U(3,4)$. Find the distribution of $V=X Y$. 
This is a simple application of the algorithm. The following Maple code defines the random variables $X$ and $Y$ and returns the PDF of their product. Note, the procedure UniformRV returns the PDF in a Maple list-of-lists data structure outlined in Glen et al. (2001):

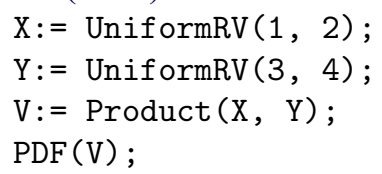

These statements yield the following PDF for $V=X Y$ :

$$
h(v)= \begin{cases}\ln v-\ln 3, & 3<v<4, \\ \ln 4-\ln 3, & 4<v<6, \\ 3 \ln 2-\ln v, & 6<v<8 .\end{cases}
$$

Note that while the PDF of both $X$ and $Y$ are defined on single segments that have positive interval limits, the PDF of $V$ is defined on three segments.

Example 4.2. Consider the random variable $X \sim \operatorname{Triangular}(1,2,3)$ and the random variable $Y \sim$ Triangular $(-2,1,2)$. Find the PDF of $V=X Y$. Since $X$ and $Y$ are each defined on two segments, this non-uniform example illustrates the case of several (four) rectangular product spaces. The APPL code in this case is:

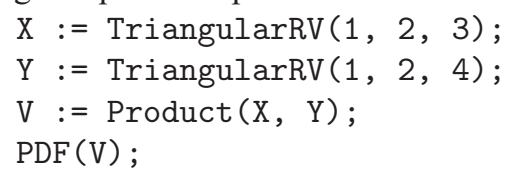

The resulting PDF for $V$ has six segments:

$$
h(v)=\left\{\begin{aligned}
-4 v / 3+(2 / 3) \ln v+(2 v / 3) \ln v+4 / 3, & 1<v<2, \\
-8+(14 / 3) \ln 2+(7 v / 3) \ln 2+10 v / 3-4 \ln v & \\
\quad-(5 v / 3) \ln v, & 2<v<3, \\
-4+(14 / 3) \ln 2+(7 v / 3) \ln 2+2 v-2 \ln v & \\
\quad-v \ln v-2 \ln 3-(2 v / 3) \ln 3, & 3<v<4, \\
44 / 3-14 \ln 2-(7 v / 3) \ln 2-8 v / 3-2 \ln 3 & \\
\quad+(22 / 3) \ln v-(2 v / 3) \ln 3+(4 v / 3) \ln v, & 4<v<6, \\
8 / 3-8 \ln 2-(4 v / 3) \ln 2-2 v / 3+(4 / 3) \ln v & \\
\quad+(v / 3) \ln v+4 \ln 3+(v / 3) \ln 3, & 6<v<8, \\
-8+8 \ln 2+(2 v / 3) \ln 2+2 v / 3+4 \ln 3 & \\
-4 \ln v+(v / 3) \ln 3-(v / 3) \ln v, & 8<v<12 .
\end{aligned}\right.
$$

Example 4.3. Consider the random variable $X \sim N(0,1)$ and the random variable $Y \sim N(0,1)$. Find the PDF of $V=X Y$. This example illustrates the case of 0 in the 


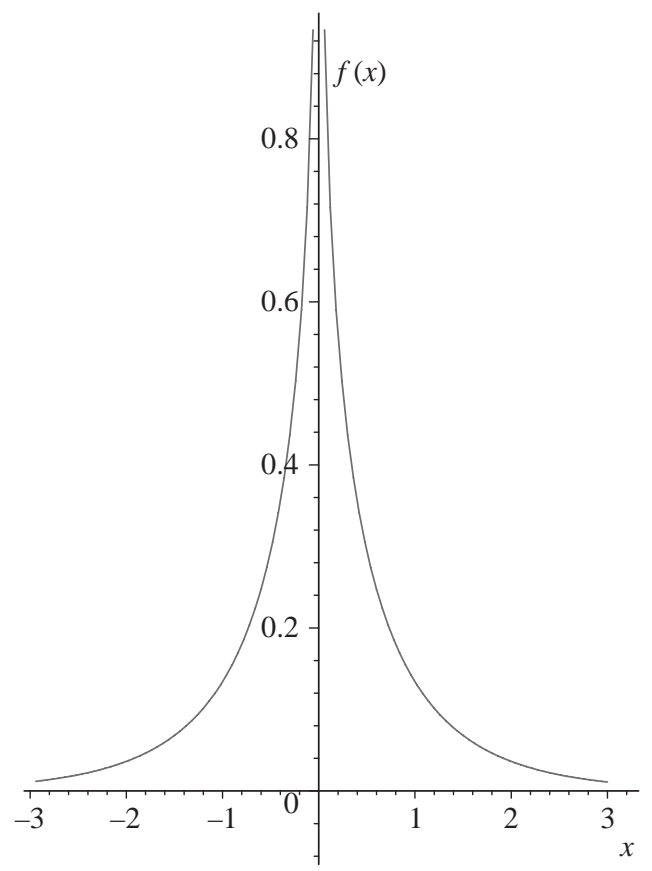

Fig. 3. The PDF of $V=X Y$ for $X \sim N(0,1)$ and $Y \sim N(0,1)$.

support of $X$ and $Y$ and also the case where the support of $X$ and $Y$ includes the endpoints $\pm \infty$.

The program yields the following PDF for $V$ :

$$
h(v)= \begin{cases}\frac{\operatorname{BesselK}(0, v \cdot \operatorname{signum}(v))}{\pi}, & -\infty<v<0, \\ \frac{\operatorname{BesselK}(0, v \cdot \operatorname{signum}(v))}{\pi}, & 0<v<\infty,\end{cases}
$$

which relies on Maple's BesselK and signum functions. An APPL plot of this function is given in Fig. 3 .

Example 4.4. Consider the independent random variables $U_{1} \sim U(0,1)$ and $U_{2} \sim U(0,1)$. The Box-Muller algorithm for generating a single standard normal deviate $V$ can be coded in one line (Devroye, 1996) as

$$
V \leftarrow \sqrt{-2 \ln U_{1}} \cos \left(2 \pi U_{2}\right),
$$

where $U_{1}$ and $U_{2}$ are independent random numbers. Using the Transform (Glen et al., 1997) and Product procedures together, one can determine the PDF of $V$. Due to the principle inverse difficulty with trigonometric functions, however, the transformation must be rewritten as

$$
V \leftarrow \sqrt{-2 \ln U_{1}} \cos \left(\pi U_{2}\right)
$$


before using Transform. The APPL code to compute the distribution of $V$ is:

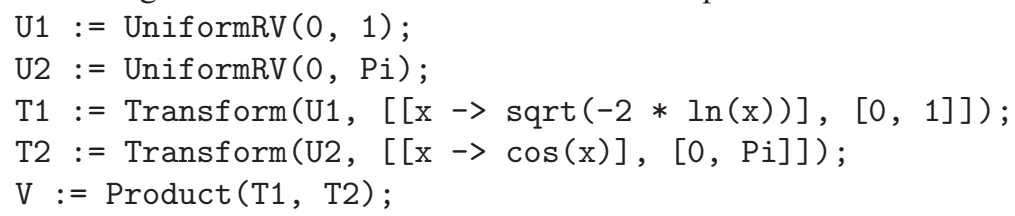

This APPL code yields the following PDF for $V$ :

$$
h(v)= \begin{cases}\frac{v}{\pi} \int_{-1}^{0} \frac{\mathrm{e}^{-v^{2} /\left(2 x^{2}\right)}}{\sqrt{1-x^{2}} x^{2}} \mathrm{~d} x, & -\infty<v<0, \\ \frac{v}{\pi} \int_{0}^{1} \frac{\mathrm{e}^{-v^{2} /\left(2 x^{2}\right)}}{\sqrt{1-x^{2}} x^{2}} \mathrm{~d} x, & 0<v<\infty .\end{cases}
$$

While this form in not easily recognizable as the PDF for the normal distribution, it is mathematically equivalent to the more standard

$$
h(v)=\frac{1}{\sqrt{2 \pi}} \mathrm{e}^{-v^{2} / 2}, \quad-\infty<v<\infty .
$$

As a final example, the Product procedure can be used in various types of statistical inference, as illustrated here in hypothesis testing.

Example 4.5 (Hogg and Craig, 1995, p. 287). Let $X_{1}$ and $X_{2}$ be iid observations drawn from a population with $\mathrm{PDF}$

$$
f(x)=\theta x^{\theta-1}, \quad 0<x<1,
$$

where $\theta>0$. Test $\mathrm{H}_{0}: \theta=1$ versus $\mathrm{H}_{1}: \theta>1$ using the test statistic $X_{1} X_{2}$ and the critical region $C=\left\{\left(X_{1}, X_{2}\right) \mid X_{1} X_{2} \geqslant 3 / 4\right\}$. Find the significance level $\alpha$ and power function for the test.

The APPL code to compute the power function is:

$\mathrm{n}:=2$;

crit $:=3 / 4$;

assume (theta $>0)$;

$\mathrm{X}:=[[\mathrm{x}->$ theta $* \mathrm{x}-($ theta -1$)],[0,1],[$ "Continuous", "PDF"] $]$;

$\mathrm{T}:=\operatorname{ProductIID}(\mathrm{X}, \mathrm{n})$;

power := SF $(\mathrm{T}$, crit);

which yields

$$
\operatorname{Pr}\left(\text { rejecting } \mathrm{H}_{0} \mid \theta\right)=1-(3 / 4)^{\theta}+\theta(3 / 4)^{\theta} \ln (3 / 4) .
$$

The fact that the population distribution is non-standard indicates that $\mathrm{X}$ must be defined using the list-of-three lists data structure shown above. The assume statement defines the parameter space. The ProductIID procedure simply makes repeated calls to Product. Finally, the SF procedure returns the survivor function, which is the complement of the CDF. To compute the significance level of the test, the additional Maple statement $\operatorname{subs}($ theta $=1$, power); 
is required, yielding $\alpha=\frac{1}{4}+\frac{3}{4} \ln \left(\frac{3}{4}\right) \cong 0.0342$. Maple's floating point evaluator evalf can be used to compute the floating point representation of $\alpha$. To plot the power function requires the additional statement:

plot (power, theta $=0 . .4$ );

Obviously, this example can be generalized for different sample sizes, population distributions, and critical values with only minor modification.

\section{Extensions}

By combining this algorithm with earlier research on automating the transformations of random variables using the transformation technique (Glen et al., 1997), it is possible to create an algorithm for finding convolutions of independent, univariate random variables. Note that the relationship $W=\ln \left(\mathrm{e}^{X} \mathrm{e}^{Y}\right)$ is the equivalent of the convolution, $W=X+Y$, when $X$ and $Y$ are independent random variables. This equivalence allows us to find the distributions of $\mathrm{e}^{X}$ and $\mathrm{e}^{Y}$, then find the distribution of the product of the two, then find the distribution of the natural logarithm of this product to yield the distribution of the convolution of $X$ and $Y$.

For example if $U_{i} \sim U(0,1)$, APPL can find the distribution of $\sum_{i=1}^{n} U_{i}$. For $n=2$, the convolution has the triangular distribution. For $n=50$, the distribution has 50 segments and takes dozens of pages of ASCII text to list the polynomials. Our current research concerning the sum of independent uniform random variables as a goodness-of-fit test statistic is promising. The statistic has higher power than the Anderson-Darling statistic, for certain cases, and has exact significance levels.

As a second example, a problem given to mathematical statistics students at the United States Military Academy requires that students model total return of investment on a portfolio as $\sum_{i=1}^{n} R_{i} T_{i}$, where the $R_{i}$ 's denote random rates and the $T_{i}$ 's denote random investment times. This problem requires students to use APPL in a way that trades off model adequacy for the component random variables (finding appropriate random variables to model rate and time) vs. model adequacy for the summation (finding random variables that will produce a usable distribution for the total return). This combination of products and convolutions can yield rather complex distributions. The result is often an un-evaluated integral, based on Maple's inability to do the necessary calculus. This problem highlights some of the limitations of the algorithm due to the base language (Maple). These limitation will be discussed in the next section.

\section{Limitations, conclusions, and further research}

Occasionally, Maple returns a PDF in a less than optimal form. The expression $\ln (-v)-I \pi$, for example, may occur as part of a PDF. Since $\ln (-1)=I \pi$, the expression is equal to $\ln v$. Also the PDF may involve a complicated integral, which Maple fails to recognize is equal to a much simpler standard form (see Example 4.4). We anticipate that future generations of computer algebra systems will overcome these difficulties. 
The algorithms that produce the product and convolutions of random variables greatly increase the "feasible" realm of random variable algebra. Summing 50 independent uniform random variables is straightforward in theory, but the implementation is not possible by hand. There are many cases, though, where the integration required by products and convolutions are intractable and thus the algorithms appear to fail. In fact, it is the underlying inability of the program (Maple) to implement the algorithm in APPL that produces the failure. In general, there are three classes of resultant distributions that come from products and convolutions via this algorithm. Class A is the set of resultant distributions that are completely tractable, such as the sum of five uniform random variables. Here the PDF is in closed form, the CDF is calculable, quantiles can be calculated, and plots of distributions are easily produced. The second set, Class B, is the set of resultant distributions that are not in closed form, but are in forms that Maple can still evaluate, such as PDFs that rely on the erf function or the BesselK functions of Maple. Example 4.3 is one such case, where the non-closed form PDF is in terms of the BesselK function, which is used in solving systems of differential equations. In Class B, it is often possible to plot the PDF and calculate quantiles. Finding a usable form for the CDF, however, might not be possible. The final class of problems, Class $\mathrm{C}$, is the set that only appears as un-evaluated integrals. The convolution of three Weibull random variables is one such example. Here the resulting PDF has little use, as Maple cannot plot the function, and cannot evaluate critical points very effectively.

In conclusion, an algorithm for calculating the PDF of the product of two independent random variables $X$ and $Y$ (which may be defined in a piece-wise manner) has been developed and implemented. The algorithm has also been extended to calculate the PDF of convolutions of independent random variables. The APPL procedure Product is one of many procedures capable of automating complicated probability calculations associated with random variables (Glen et al., 2001). Potential applications for calculations of this type occur in all areas of applied probability and statistics.

\section{Acknowledgements}

The authors gratefully acknowledge the assistance of Professor Hank Krieger in the early work associated with this paper and Professor Donald Barr for his assistance throughout the project. The authors acknowledge the helpful comments from the reviewers and Professor Diane Evans. The second author acknowledges support from the The College of William and Mary's Faculty Research Assignment program.

\section{Appendix}

This appendix gives the details associated with the algorithm that computes the distribution of the product of two continuous, independent random variables. The algorithm has been implemented in Maple and is one of the procedures in APPL. Cardinality is denoted by $\|\cdot\|$ and the empty list is denoted by [ ]. Indentation is used to indicate 
loops and conditions. The conditions prior to the calculation of the integrals are to avoid calculating integrals unnecessarily.

\section{Procedure product}

Input: The distribution of the random variable $X$ and the distribution of the random variable $Y$, where $X$ and $Y$ are independent, univariate, continuous random variables. The PDFs are in the list-of-lists format, i.e., the random variable $X$ is represented by the data structure $\left[\tilde{f}(x), x^{\star},[\right.$ "Continuous", "PDF"]], and the random variable $Y$ is represented by the data structure $\left[\tilde{g}(y), y^{\star}\right.$, ["Continuous", "PDF"]], as described in Glen et al. (2001). The first sublist for $X$ contains a list of the piece-wise $f(x)$ functions, that is, $\tilde{f}(x)=\left[f_{1}(x), f_{2}(x), \ldots, f_{n}(x)\right]$. The second sublist for $X$ contains a list of the endpoints of the support of $X$, that is, $x^{\star}=\left[x_{1}^{\star}, x_{2}^{\star}, \ldots, x_{n+1}^{\star}\right]$, where $f(x)=f_{i}(x)$ for $x_{i}^{\star}<x<x_{i+1}^{\star}, i=1,2, \ldots, n$.

Output: The PDF of $V=X Y, h(v)$, in the same list-of-lists format as the input. The list $\tilde{h}(v)=\left[h_{1}(v), h_{2}(v), \ldots, h_{l}(v)\right]$ contains the piece-wise elements of the PDF of $V$ and $v^{\star}=\left[v_{1}^{\star}, v_{2}^{\star}, \ldots, v_{l+1}^{\star}\right]$ contains the endpoints of the support of $V$. The PDF is determined by a special case of Rohatgi's result for two independent random variables

$$
h(v)=\int_{-\infty}^{\infty} f(x) g\left(\frac{v}{x}\right) \frac{1}{|x|} \mathrm{d} x .
$$

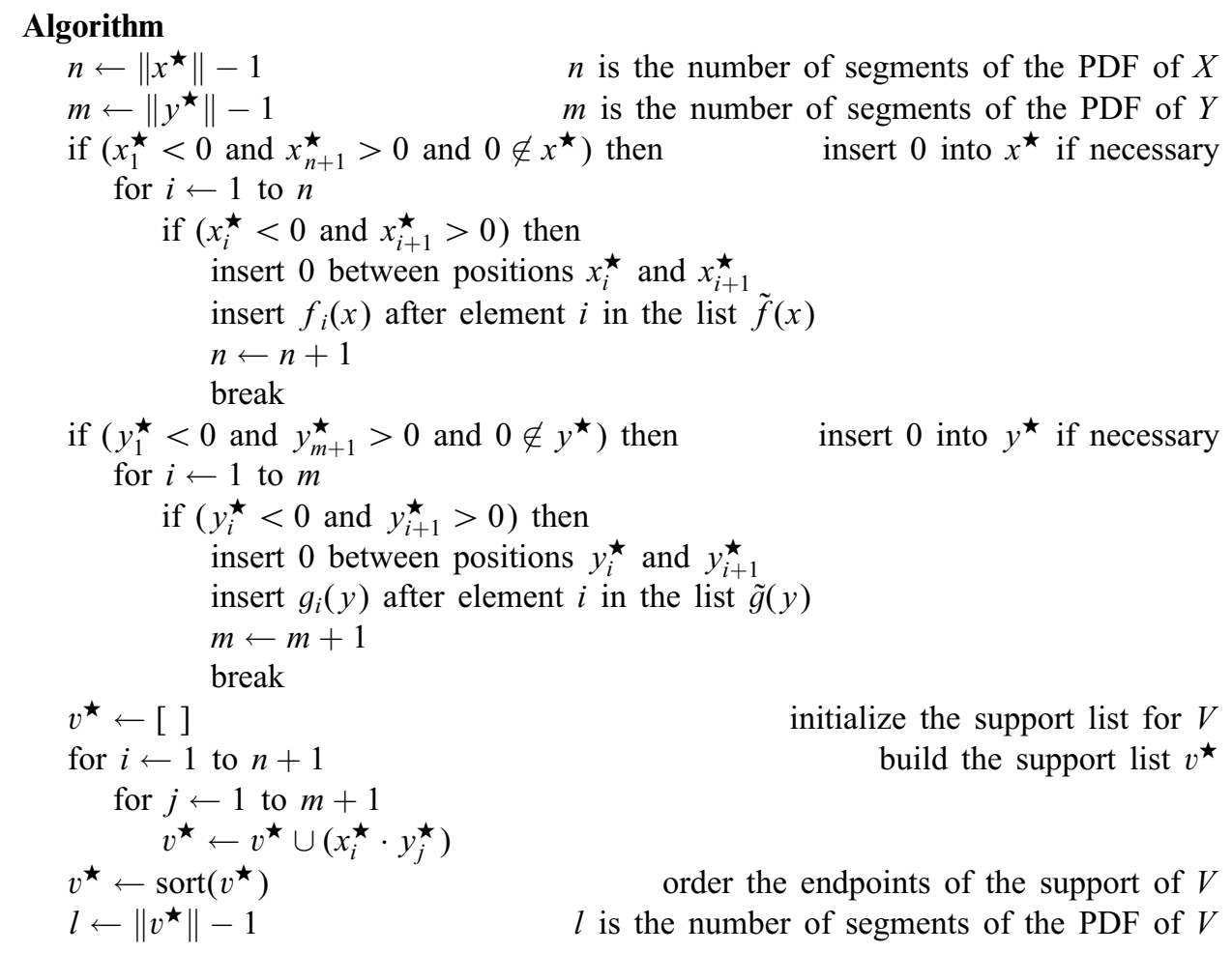




$$
\begin{gathered}
\text { for } i \leftarrow 1 \text { to } l \\
h_{i}(v) \leftarrow 0
\end{gathered}
$$$$
\text { for } i \leftarrow 1 \text { to } n
$$$$
\text { for } j \leftarrow 1 \text { to } m
$$$$
\begin{aligned}
& a \leftarrow x_{i}^{\star} \\
& b \leftarrow x_{i+1}^{\star} \\
& c \leftarrow y_{j}^{\star} \\
& d \leftarrow y_{j+1}^{\star} \\
& \text { if }(a \geqslant 0 \text { and } c \geqslant 0) \text { then } \\
& \quad p(v) \leftarrow \int_{a}^{b} f_{i}(x) \cdot g_{j}\left(\frac{v}{x}\right) \cdot \frac{1}{x} \mathrm{~d} x
\end{aligned}
$$

initialize PDF segments of $V$

loop through each piecewise segment of $X$ loop through each piecewise segment of $Y$ define corners of the rectangular region

the region is in the first quadrant compute integrals required subsequently

if $(d<\infty)$ then $q(v) \leftarrow \int_{v / d}^{b} f_{i}(x) \cdot g_{j}\left(\frac{v}{x}\right) \cdot \frac{1}{x} \mathrm{~d} x$

if $(c>0)$ then $r(v) \leftarrow \int_{a}^{v / c} f_{i}(x) \cdot g_{j}\left(\frac{v}{x}\right) \cdot \frac{1}{x} \mathrm{~d} x$

if $(c>0$ and $d<\infty$ and $a d<b c)$ then $s(v) \leftarrow \int_{v / d}^{v / c} f_{i}(x) \cdot g_{j}\left(\frac{v}{x}\right) \cdot \frac{1}{x} \mathrm{~d} x$

if $(c=0$ and $d=\infty)$ then

Scenario A for $k \leftarrow 1$ to $l$

if $\left(v_{k}^{\star} \geqslant 0\right)$ then $h_{k}(v) \leftarrow h_{k}(v)+p(v)$

if $(c=0$ and $d<\infty)$ then

Scenario B

for $k \leftarrow 1$ to $l$

if $\left(v_{k}^{\star} \geqslant 0\right.$ and $\left.v_{k+1}^{\star} \leqslant a d\right)$ then $h_{k}(v) \leftarrow h_{k}(v)+p(v)$

if $\left(v_{k}^{\star} \geqslant a d\right.$ and $\left.v_{k+1}^{\star} \leqslant b d\right)$ then $h_{k}(v) \leftarrow h_{k}(v)+q(v)$

if $(c>0$ and $d=\infty)$ then

Scenario C

for $k \leftarrow 1$ to $l$

if $\left(v_{k}^{\star} \geqslant b c\right)$ then $h_{k}(v) \leftarrow h_{k}(v)+p(v)$

if $\left(v_{k}^{\star} \geqslant a c\right.$ and $\left.v_{k+1}^{\star} \leqslant b c\right)$ then $h_{k}(v) \leftarrow h_{k}(v)+r(v)$

if $(c>0$ and $d<\infty)$ then

if $(a d<b c)$ then

for $k \leftarrow 1$ to $l$

if $\left(v_{k}^{\star} \geqslant a c\right.$ and $\left.v_{k+1}^{\star} \leqslant a d\right)$ then $h_{k}(v) \leftarrow h_{k}(v)+r(v)$

if $\left(v_{k}^{\star} \geqslant a d\right.$ and $\left.v_{k+1}^{\star} \leqslant b c\right)$ then $h_{k}(v) \leftarrow h_{k}(v)+s(v)$

if $\left(v_{k}^{\star} \geqslant b c\right.$ and $\left.v_{k+1}^{\star} \leqslant b d\right)$ then $h_{k}(v) \leftarrow h_{k}(v)+q(v)$

if $(a d=b c)$ then

for $k \leftarrow 1$ to $l$

if $\left(v_{k}^{\star} \geqslant a c\right.$ and $\left.v_{k+1}^{\star} \leqslant a d\right)$ then $h_{k}(v) \leftarrow h_{k}(v)+r(v)$

if $\left(v_{k}^{\star} \geqslant b c\right.$ and $\left.v_{k+1}^{\star} \leqslant b d\right)$ then $h_{k}(v) \leftarrow h_{k}(v)+q(v)$

if $(a d>b c)$ then

for $k \leftarrow 1$ to $l$

if $\left(v_{k}^{\star} \geqslant a c\right.$ and $\left.v_{k+1}^{\star} \leqslant b c\right)$ then $h_{k}(v) \leftarrow h_{k}(v)+r(v)$

if $\left(v_{k}^{\star} \geqslant b c\right.$ and $\left.v_{k+1}^{\star} \leqslant a d\right)$ then $h_{k}(v) \leftarrow h_{k}(v)+p(v)$

if $\left(v_{k}^{\star} \geqslant a d\right.$ and $\left.v_{k+1}^{\star} \leqslant b d\right)$ then $h_{k}(v) \leftarrow h_{k}(v)+q(v)$

Scenario D

Case I

Case II

Case III

execute analogous steps for rectangles in quadrants II, III, and IV remove any redundancies in $h(v)$ due to 0 in the support list return $\left(\left[\tilde{h}(v), v^{*}\right.\right.$, ["Continuous", "PDF"]]). 


\section{References}

Devroye, L., 1996. Random variate generation in one line of code. In: Charnes, J., Morrice, D., Brunner, D., Swain, J. (Eds.), Proceedings of the 1996 Winter Simulation Conference. Institute of Electrical and Electronics Engineers, Coronado, CA, Institute of Electrical and Electronics Engineers, Piscataway, NJ, pp. $265-272$.

Glen, A., Leemis, L., Drew, J., 1997. A Generalized univariate change-of-variable transformation technique. INFORMS J. Comput. 9 (3), 288-295.

Glen, A., Evans, D., Leemis, L., 2001. APPL: a probability programming language. Amer. Statist. 55 (2), $156-166$.

Hogg, R.V., Craig, A.T., 1995. Mathematical Statistics, 5th Edition. Prentice-Hall, Englewood Cliffs, NJ. Maple Version 7, 2001. Waterloo Maple, Inc. Waterloo, Canada. Rohatgi, V.K., 1976. An Introduction to Probability Theory Mathematical Statistics. Wiley, New York. Springer, M.D., 1979. The Algebra of Random Variables. Wiley, New York. 